\title{
Recanalisation of cerebral artery aneurysms treated endovascularly - a midterm follow-up
}

\author{
Witold Tomalski ${ }^{1}$, Daniel Knap ${ }^{2}$, Amadeusz Żak ${ }^{3}$, Łukasz Binek ${ }^{2}$, \\ Milena Dewerenda-Sikora ${ }^{2}$, Aleksandra Krzan², Przemysław Puz ${ }^{3}$, Maciej Tomalski ${ }^{4}$, \\ Dominik Sieron ${ }^{5}$, Wojciech Piwowarski ${ }^{1}$, Wacław Kuczmik ${ }^{6}$, Anetta Lasek-Bal ${ }^{3}$ \\ ${ }^{1}$ Department of Neurosurgery, Upper-Silesian Medical Centre of the Silesian Medical University in Katowice, Poland \\ ${ }^{2}$ Upper-Silesian Medical Centre of the Silesian Medical University in Katowice, Poland \\ ${ }^{3}$ Department of Neurology, School of Health Sciences, Medical University of Silesia in Katowice, Katowice, Poland \\ ${ }^{4}$ Medical University of Silesia in Katowice, Poland \\ ${ }^{5}$ Institute of Radiology, Tiefenau Hospital, Inselgroup, University of Bern, Switzerland \\ ${ }^{6}$ Department of General Surgery, Vascular Surgery, Angiology and Phlebology, Medical University of Silesia in Katowice, Poland
}

\begin{abstract}
Endovascular methods of aneurysm treatment, as an alternative to neurosurgical clipping, have proved a welcome opportunity to treat patients with unruptured aneurysms or those disqualified from neurosurgical intervention. This paper presents our own experience of endovascular treatment of cerebral aneurysms in 107 patients. It includes clinical and technical data from the perioperative period and a 12-month radiological follow-up of 78 patients.

Method. Our retrospective evaluation covered patients with intracranial aneurysms treated endovascularly. The following were analysed: age, sex, neurological symptoms, and familial burden of intracranial aneurysm. Multivariate analysis was performed to determine independent factors of recanalisation of the cerebral aneurysm 12 months after embolisation.

Results. The data of 107 patients at a mean age of 61 years [57.09 \pm 14.27$]$ treated with embolisation was analysed. The indication for intervention in 16 patients was subarachnoid haemorrhage; in the remaining 91 cases, aneurysms were revealed during diagnostic procedures for different symptoms or during imaging examinations. The intracranial segment of the internal carotid artery and the anterior communicating artery were the most common locations for aneurysms. After embolisation, subarachnoid haemorrhage occurred in one patient, ischaemic stroke in two patients, and one patient died because of acute circulatory insufficiency. The functional status of 94 patients on the day of discharge from the department (on days 4-21) was very good. 78 patients completed a 12-month follow-up period. In 11 of those, a follow-up MR angiography revealed recanalisation 12 months after the intervention. Except for one patient reporting vertigo, aneurysm recanalisation procedures were asymptomatic. The only independent risk factor for recanalisation was the size of aneurysm $>10 \mathrm{~mm} ; \mathrm{OR} \mathrm{3.0;} \mathrm{CI} \mathrm{[1.15-7.83]} \mathrm{p}=0.0255$.

Conclusions. Embolisation of cerebral aneurysms is a safe method with few perioperative complications, and most of these are mild and transient. The size of the aneurysm during qualification for embolisation is a risk factor for recanalisation in the subsequent 12 months. Recanalisation of embolised cerebral aneurysms concerns less than $20 \%$ of patients in a one-year follow-up and is most often asymptomatic.
\end{abstract}

Key words: cerebral aneurysm, embolisation, recanalisation

(Neurol Neurochir Pol 2020; 54 (6): 524-530)

Address for correspondence: Przemysław Puz, Department of Neurology, School of Health Sciences, Medical University of Silesia in Katowice, Ziołowa 45/47, 40-635 Katowice, Poland, e-mail: ppuz@o2.pl 


\section{Background}

Intracranial aneurysms, assessed on the basis of post mortem examinations, are estimated to affect $1-5 \%$ of the adult population [1]. They are reported three times more frequently in women than in men [2]. Most cerebral aneurysms do not rupture. Improved access to neuroimaging examinations has enabled physicians to find more intracranial vascular abnormalities prior to the appearance of neurological symptoms. Endovascular methods of aneurysm treatment, as an alternative to neurosurgical clipping, have proved a welcome opportunity for patients with unruptured aneurysms and have helped those disqualified from neurosurgical intervention. Compared to surgical clipping of aneurysms, endovascular therapy is substantially less invasive [3]. The risk of aneurysm rupture depends on the patient's clinical profile (i.e. age, location, familial burden of aneurysms) and the morphological features of that aneurysm (size and shape) [3]. Before deciding about the indication for a specific treatment and before the choice of method, the risk of natural course (i.e. will the aneurysm rupture if no intervention is performed) is compared against the risk related to the particular neurosurgical or endovascular procedure.

There are these days many different techniques used in endovascular treatment of cerebral aneurysms, e.g. simple coiling, balloon remodelling, stent remodelling, 2-stent remodelling in various configurations, flow diversion, and intrasaccular flow disruption, as well as combinations of the abovementioned methods.

The choice of a specific treatment technique depends on a number of factors, i.e.: the morphology of the aneurysm and in particular the width of the aneurysm neck, the preference of the operator, the availability of equipment in the laboratory, and the anatomy of the arteries feeding the aneurysm [4]. Aneurysm recanalisation after endovascular treatment affects up to one third of patients, usually within the 12 months following intervention. The risk of recanalisation depends on the parameters related to the morphology of the aneurysm and the technical aspects of the procedure.

This study looks at the effectiveness and safety of embolisation of ruptured and unruptured cerebral artery aneurysms.

\section{Method}

This paper presents our own experience with endovascular treatment of mostly unruptured cerebral aneurysms in 107 patients exhibiting clinical and technical data from the perioperative period and at least a 12 -month radiological follow-up of 78 patients (72.89\% of the 107).

Retrospective evaluation involved 108 patients who, between January 2017 and December 2019, were hospitalised in the Neurology Department of the Upper-Silesian Medical Centre of the Silesian Medical University in Katowice, Poland for embolisation of ruptured or unruptured intracranial aneurysms with the use of coils and/or stents. Preliminary diagnosis of an aneurysm was established on the basis of head computed tomography (CT) or magnetic resonance (MR) angiography (CTA, MRA).

The following data was analysed: the age and the sex of the patient qualified for embolisation, their neurological symptoms, any other diseases, and any family history of intracranial aneurysm. Moreover, the parameters related to aneurysm, i.e. size, location, rupture and individual history, were analysed for the presence of other aneurysms and previous endovascular procedures. For the description of parametric data, the mean and standard deviation, median, minimum and maximum values of a given variable in the study group were calculated. Multivariate analysis was performed to determine independent factors of recanalisation (based on MR angiography) of the cerebral aneurysm 12 months after embolisation. The following parameters were analysed: age, sex, arterial hypertension, diabetes, dyslipidemia and the size of the aneurysm (i.e. $<10 \mathrm{~mm}, 10-24 \mathrm{~mm}$, or $>24 \mathrm{~mm}$ ).

Description of cerebral aneurysm embolisation

All patients undergoing diagnostic procedures had a digital subtraction angiography (DSA) performed using X-ray (Siemens Axiom Artis dTa) as per the defined protocols. The other part of the examination involved the use of a DSA workstation for further image analysis. Aneurysms were analysed at the DSA station based on the imaging results. The following features were assessed:

- location;

- aneurysm sac dimensions - the longest dimension of length, height, and width;

- aneurysm neck dimension - the diameter measured in two extreme dimensions;

- sac-to-neck ratio - the longest sac dimension and the longest neck dimension were used for calculations; these were divided into three groups: ratio $<1.2$; between 1.2 and 1.5 ; and $>1.5$ [5];

- aneurysm size - the aneurysms were divided by size: small aneurysm $<10 \mathrm{~mm}$ in diameter; large aneurysm $10-24 \mathrm{~mm}$; or giant aneurysm $>25 \mathrm{~mm}[6,7]$. The assessment of the aneurysm sac is presented in Figure 1.

Depending on the sac-to-neck ratio, the aneurysms were treated using the following techniques:

a) simple coiling;

b) balloon remodelling;

c) stent remodelling using:

- a single stent,

- or two stents in $\mathrm{Y}, \mathrm{X}$ or $\mathrm{T}$ configurations;

d) flow diversion.

The coils were implanted into the aneurysm sac using a micro-catheter and they were detached while using fluoroscopic viewing. The coils were introduced into the aneurysm sac until the aneurysm was filled up and no more coils could be inserted, or until no further inflow of the contrast agent into the aneurysm sac could be seen in angiography. 


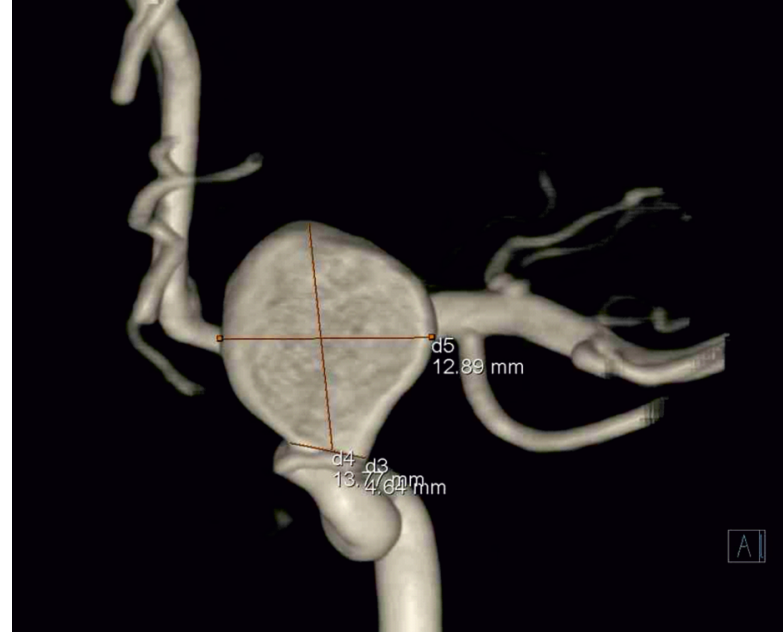

Figure 1A. Left internal carotid artery aneurysm in the ophthalmic segment (C6) - the sac dimension (length and height)

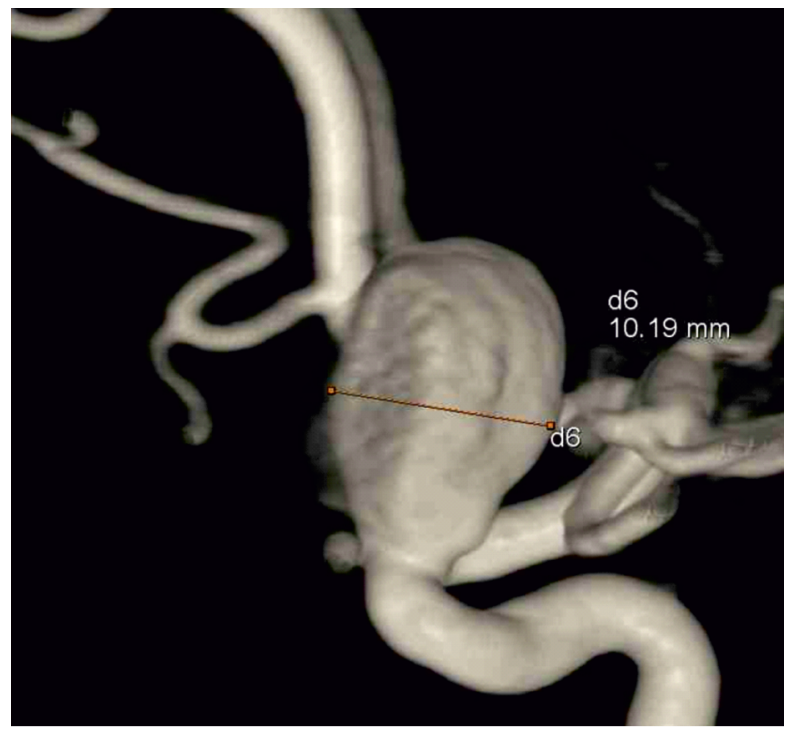

Figure 1B. Left internal carotid artery aneurysm in the ophthalmic segment (C6) - the sac dimension (width)

Stent embolisation was mainly applied in patients with wide-neck aneurysms. The most commonly used was the hybrid stent (Atlas by Stryker) and the second most commonly used was the braided stent (Leo, Leo Baby by Balt). Flow diverters (Silk by Balt) were used in small quantities and only if no other endovascular technique was suitable for the given aneurysm. A stent was implanted to cover the aneurysm neck; if that aneurysm was located at an artery branching, we chose the branch which enabled us to cover as much of the aneurysm neck as possible. This technique often requires catheterisation of the M2, A2 branches that start at an unfavourable angle of the aneurysm neck. In such cases, a combination of pre-shaped (S, J, C) Sl10 microcatheters (Stryker) with a Hybrid 008J micro guidewire (Balt) was successfully applied. Due to the unique properties of a hybrid stent, a 2-stent Y or T-configuration placement was very rarely necessary.

Those patients who were planned for stent implantation received pretreatment with antiplatelet agents. On the day before the procedure, the patient was given $600 \mathrm{mg}$ of clopidogrel and $300 \mathrm{mg}$ of aspirin. Patients who underwent stent implantation into the cerebral arteries were administered $75 \mathrm{mg}$ of clopidogrel for three months and $150 \mathrm{mg}$ of aspirin daily for six months following stent placement. Follow-up head MR angiography was scheduled for 12 months post intervention. Residual flow was classified as: A) total obliteration; B) contrast-filled neck without sac-filling; or C) residual aneurysm with sac-filling.

\section{Results}

There were 108 patients with a mean age of 61 years [57.09 \pm 14.27$]$ qualified for embolisation. In one patient with an unruptured aneurysm, intervention was abandoned for technical reasons (after angiography), meaning that eventually data from 107 patients was collected and analysed. Half of the patients were aged $50-70$ and nearly $80 \%$ of the whole group were female. The indication for intervention in $16 \mathrm{pa}-$ tients (15\%) was subarachnoid haemorrhage in the course of aneurysm rupture; in the remaining cases, aneurysms were revealed during diagnostic procedures for headaches (most commonly) or during imaging examinations unrelated to neurological symptoms. The intracranial segment of the internal carotid artery and the anterior communicating artery were the most common locations for aneurysms. Nearly one in four of the patients revealed another intracranial aneurysm; one in 10 patients had previously undergone endovascular therapy due to an aneurysm in another location. The great majority of patients (73\%) underwent small aneurysm embolisation (small being defined where the largest aneurysm was $<10 \mathrm{~mm}$ ).

Clinical and radiological description of the patients who underwent embolisation is presented in Table 1.

In 51 patients (53.76\%), at least six coils were implanted, and stents were placed in 45 patients (42.05\%). The most common symptoms reported by the patients in the 72 hours following intervention were: diffuse headache, vertigo, and visual disturbances. Subarachnoid haemorrhage occurred in one patient. Ischaemic stroke occurred in two patients. One patient with a ruptured ACoA aneurysm died due to acute circulatory insufficiency.

The functional status of nearly $90 \%$ of patients on the day of discharge from the department (on days 4-21) was very good. Those patients who required caregiver assistance were previously qualified for embolisation due to a ruptured cerebral aneurysm. Table 2 presents the parameters in the perioperative period.

Seventy-eight $(72.89 \%)$ patients completed a 12-month follow-up period. In 11 of those (14.10\%), a follow-up MR angiography revealed recanalisation after 12 months. The degree 
Table 1. Characteristics of patients undergoing cerebral aneurysm embolisation

\begin{tabular}{|c|c|}
\hline Parameter & $\mathbf{N}(\%)$ \\
\hline Mean age [range] & $61[25-82]$ \\
\hline$<30$ & $5(4.67)$ \\
\hline $30-49$ & $29(27.1)$ \\
\hline $50-69$ & $55(51.4)$ \\
\hline$\geq 70$ & $18(16.51)$ \\
\hline $\mathrm{F}$ & $83(77.57)$ \\
\hline Arterial hypertension & $61(57)$ \\
\hline Diabetes & $8(7.48)$ \\
\hline Dyslipidemia & $30(28.03)$ \\
\hline \multicolumn{2}{|l|}{ Neurological signs before qualification for embolisation: } \\
\hline Headaches & $46(58.97)$ \\
\hline Vertigo & $31(39.74)$ \\
\hline Visual disturbances & $6(7.68)$ \\
\hline Tinnitus & $20(25.64)$ \\
\hline Seizures & $3(3.84)$ \\
\hline No complaints & $23(29.48)$ \\
\hline Cerebral aneurysm in a family member & $2(2.56)$ \\
\hline \multicolumn{2}{|l|}{ Aneurysm location: } \\
\hline $\mathrm{ACOA}$ & $17(15.89)$ \\
\hline MCA & $14(13.08)$ \\
\hline BA & $10(9.35)$ \\
\hline ICA intracranially & $53(49.53)$ \\
\hline ACA & $4(3.74)$ \\
\hline VA & $3(2.8)$ \\
\hline \multicolumn{2}{|l|}{ Lateralisation (without ACoA) } \\
\hline L & $39(50.65)$ \\
\hline Ruptured aneurysm & $16(14.95)$ \\
\hline Hunt and Hess Scale score & 3 \\
\hline I & 5 \\
\hline ॥ & 5 \\
\hline III & 3 \\
\hline \multicolumn{2}{|l|}{ IV } \\
\hline Presence of other cerebral aneurysms & $24(22.43)$ \\
\hline Previous procedure for (another) cerebral aneurysm & $12(11.21)$ \\
\hline
\end{tabular}

$\mathrm{F}$ - female; $\mathrm{ACOA}$ - anterior communicating artery; $\mathrm{MCA}$ - middle cerebral artery; $\mathrm{BA}$ - basilar artery; ICA - internal carotid artery; ACA — anterior cerebral artery; VA — vertebral artery

of recanalisation in 10 patients was defined as grade $\mathrm{B}$, and in one patient as grade $\mathrm{C}$. This grade $\mathrm{C}$ patient required management during reintervention. For the remaining patients, a check-up using MR angiography at six months was recommended. Except for one patient reporting vertigo (probably of multifactorial aetiology), the patients requiring a second procedure due to aneurysm recanalisation were asymptomatic.
Table 2. Parameters related to cerebral aneurysm embolisation in 78 patients undergoing a follow-up of at least 12 months

\begin{tabular}{|c|c|}
\hline Parameter & N (\%) \\
\hline \multicolumn{2}{|l|}{ Aneurysm size* } \\
\hline$<10 \mathrm{~mm}$ & $78(72.89)$ \\
\hline $10-24 \mathrm{~mm}$ & $28(26.16)$ \\
\hline$>24 \mathrm{~mm}$ & $1(0.94)$ \\
\hline \multicolumn{2}{|l|}{ Number of coils ${ }^{* *}$} \\
\hline$<6$ & $43(45.74)$ \\
\hline$\geq 6$ & $51(54.26)$ \\
\hline Stent implantation & $45(42.05)$ \\
\hline \multicolumn{2}{|l|}{ Antiplatelet therapy: } \\
\hline ASA monotherapy & $34(31.77)$ \\
\hline CLP monotherapy & $1(0.93 \%)$ \\
\hline Duotherapy & $53(49.53)$ \\
\hline \multicolumn{2}{|c|}{$\begin{array}{l}\text { Complications / clinical status of patients, } 1-3 \text { days } \\
\text { after intervention: }\end{array}$} \\
\hline Haematoma at puncture site & $5(4.67)$ \\
\hline Headaches & $11(10.28)$ \\
\hline Vertigo / visual disturbances & $3(2.8)$ \\
\hline Ischaemic stroke & $2(1.87)$ \\
\hline Death & $1(0.93)$ \\
\hline SAH & $1(0.93)$ \\
\hline Heart failure & $3(2.8)$ \\
\hline \multicolumn{2}{|c|}{ Functional status on day of discharge on mRS: } \\
\hline $0-2$ & $94(87.85)$ \\
\hline $3-5$ & $12(11.21)$ \\
\hline Death during hospitalisation & $1(0.93)$ \\
\hline
\end{tabular}

ASA - acetylsalicylic acid; CLP - clopidogrel; SAH — subarachnoid haemorrhage; mRS - modified Rankin Scale; *based on head MR angiography; ${ }^{* *}$ data from 94 procedures

The only independent risk factor for recanalisation was the size of aneurysm $>10 \mathrm{~mm}$ (Tab. 3 ).

The internal carotid artery aneurysm before and after embolisation is presented in Figure 2.

\section{Discussion}

The most important conclusion that we draw from our study is that cerebral aneurysm embolisation is a safe method. Recanalisation affects $14 \%$ of patients 12 months following the intervention. The great majority of patients were asymptomatic and did not require urgent management. Instead, radiological follow-up was indicated.

Anterior intracranial vascular supply proved to be the most common location of aneurysms qualified for endovascular therapy. This accords with the findings of epidemiological studies related to the location of cerebral aneurysms. In our group of patients, diseases causing angiopathy (arterial 
Table 3. Risk of recanalisation of aneurysm 12 months after embolisation*

$\begin{array}{lcc} & \text { p (factor) } & \text { RR } \\ \text { Size of aneurysm } & 0.0255 & 3.0 \\ >10 \mathrm{~mm} & & 95 \% \mathrm{Cl}[1.15-7.83]\end{array}$

*The following co-variables were analysed: age, sex, diabetes, arterial hypertension, dyslipidemia, and size of aneurysm $\leq 10 \mathrm{~mm}$ or $>10 \mathrm{~mm}$

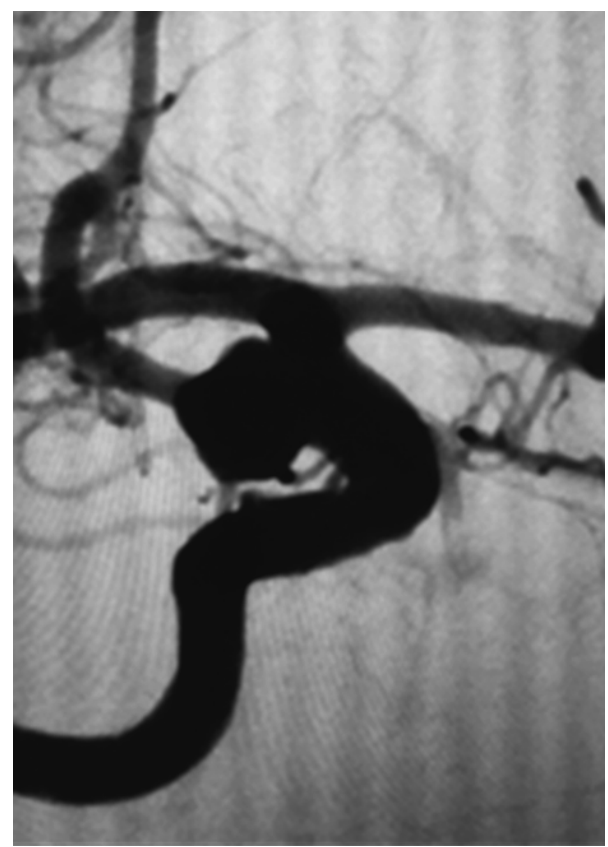

Figure 2A. Right internal carotid artery aneurysm with a wide neck before embolisation

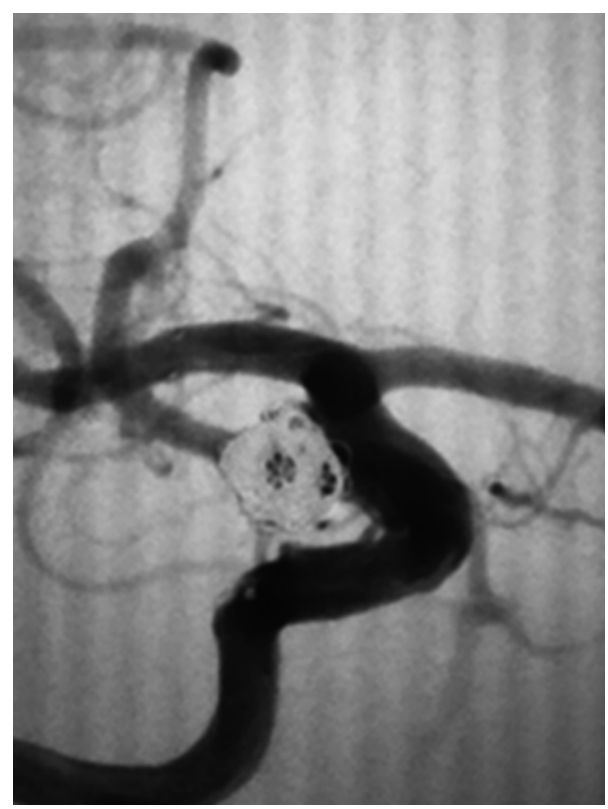

Figure 2B. Right internal carotid artery aneurysm with a wide neck after embolisation with stent securing the aneurysm neck and coils hypertension, dyslipidemia) were common in $60 \%$ and $28 \%$, respectively. Women made up the majority of embolised patients, which is consistent with the gender distribution among patients with cerebral aneurysms [2].

The most common symptoms reported by patients within the first few days following the intervention were headaches with vertigo and atypical visual disturbances. Considering the type and transient nature of the complaints, these probably resulted from vasoconstriction. Two patients suffered ischaemic stroke in the peri-interventional period; one had a subarachnoid haemorrhage. Inguinal haematoma was found in $5 \%$ of patients and this was the most frequent extra-neurological complication of the procedure. One patient died during hospitalisation due to acute circulatory insufficiency. The functional status of the great majority of patients on the day of discharge was very good. According to the literature, the most common complications after embolisation of cerebral aneurysms are haematoma in the groin, false aneurysm, and infection. Less frequently reported are arterial dissection (0.7\%), embolism (2.5\%), and arterial occlusion (2\%) [8]. According to observations reported by other authors, the incidence of ischaemic stroke in the perioperative period is higher than that of intracranial haemorrhage: $4.81 \%$ vs $1.87 \%$, respectively (National Inpatient Sample Database) [9]. The mechanism of ischaemia in this group of patients may have multiple origins. Hypercoagulability status plays a significant role, along with the following: inappropriate anticoagulation before and during intervention, unstable atherosclerotic plaque, and coil extension into the proximal artery $[10,11]$. The risk of thrombosis increases if the stent does not appose well to the vessel wall and/or two stents are used (especially in the Y or $\mathrm{X}$ configurations). Endothelial damage during the procedure may stimulate thrombus formation. Cerebral stroke is more likely to occur when managing large aneurysms. The size of the thrombus is a risk factor for its propagation and/or distal embolisation. The above-mentioned complications are reported more often in relation to previously clipped aneurysms than to coiled aneurysms $[9,12]$.

In accordance with the results of meta-analysis of data from 7,172 patients, poor functional condition/death is observed in $4.7 \%$ of patients undergoing embolisation [13]. A significantly higher percentage of patients with a good therapeutic outcome was found among those treated after 2004 (the analysis related to the period 2000 to 2011). A higher risk of poor functional status was demonstrated after treatment with liquid embolic agents (8.1\%; 99\% CI: 4.7\%, 13.7\%) than after coil placement (4.9\%; 99\% CI: 3.8\%, 6.3\%; p = 0.002) and the highest risk was demonstrated after treatment with flow diversion (11.5\%; 99\% CI: 4.9\%, 24.6\%) [14].

In the BRAT study, embolisation was associated with a higher percentage of patients in good functional status than in those after microsurgical clip occlusion in a 1.3 and 6-year observation, particularly in the analysis of the effects of 
aneurysm treatment in the posterior part of cerebral circulation [14-16]. However, analysis of the results 10 years post intervention did not reveal any significant differences in this respect $[17,18]$. The results clearly proved significantly more frequent recanalisation after endovascular therapy: $16.4 \% \mathrm{vs}$ $4.6 \%(\mathrm{p}<0.0001)$ after a 6-year follow-up [16].

The incidence of recanalisation after embolisation of cerebral aneurysms varies between $5 \%$ and $34 \%$ [19]. The risk of recanalisation of an aneurysm depends on the parameters related to its morphology (shape/size) and the technique applied for the procedure $[19,20]$. This risk is higher when the aneurysm sac size is $>10 \mathrm{~mm}$ and/or neck size is $>4 \mathrm{~mm}$ [5]. In our study, the size of the aneurysm was the only independent risk factor for recanalisation. The results of one study showed that an aneurysm sac $>10 \mathrm{~mm}$ multiplies the risk of recanalisation $\times 4.5$ times [21]. It is probable that a larger aneurysm volume makes it more difficult to stabilise and increase the density of coil placement, thus making it easier for coils to slide out of their original positions. According to many authors, coil filling density of $20-25 \%$ protects against recanalisation [6,8]. In our presented paper, 5/11 patients after recanalisation had an aneurysm sized 10-24 mm; seven of them had at least six coils implanted. According to the published data, recanalisation is observed in $35 \%$ of patients with $11-25 \mathrm{~mm}$ aneurysms, and in as many as $59 \%$ of patients with giant aneurysms (> $25 \mathrm{~mm}$ ) after embolisation [8].

One of our patients had a giant aneurysm, and in that case recanalisation also occurred.

Evaluation of haemodynamic parameters in the aneurysm sac (i.e. flow velocity and wall shear stress) indicates a significantly lower amplitude reduction of the velocity on the neck plane in patients undergoing recanalisation $[22,23]$. The role of inflammation in the recanalisation process is not yet established, which is probably important bearing in mind other factors in the process of formation, progression and rupture of aneurysms [24,25].

According to some authors, the differences in the incidence of recanalisation result from the interval between the intervention and follow-up examination. In most study sites, it is recommended that patients undergo a follow-up every six months (usually involving a head MR angiography). Given the low probability of delayed recanalisation, careful monitoring is still warranted, although at less frequent intervals (every 2-3 years) beyond 36 months post embolisation [26]. Some observations have suggested that the radiological condition reported six months after embolisation remains stable for at least two years [27].

According to studies carried out by Canadian authors, most recanalised aneurysms are visualised in MR angiography within a follow-up period of 12.31 months [28].

Recanalisation is most often asymptomatic. In this case, a routine radiological check-up allows for the assessment of the long-term effects of the intervention.

\section{Limitations}

We are aware of the limitations of our study, the most significant being its retrospective nature. No doubt the use of DSA angiography to assess the degree of recanalisation would be a more valuable procedure. However, due to the invasiveness of the examination and its limited availability, we decided to adopt head MR angiography instead.

\section{Conclusions}

Embolisation of cerebral aneurysms is a safe method with few perioperative complications, most of them being mild and transient.

The size of the aneurysm during qualification for embolisation is a risk factor for recanalisation in the subsequent 12 months.

Recanalisation of embolised cerebral aneurysms afflicts fewer than one in five patients in a 12-month follow-up, and is most often asymptomatic.

\section{References}

1. Connolly ES, Solomon RA. Management of unruptured aneurysms. Le Roux PD, Winn HR, Newell DW (Eds.). Management of Cerebral Aneurysms. Saunders, Philadelphia, Pa, USA. ; 2004: 271-285.

2. Ajiboye N, Chalouhi N, Starke RM, et al. Unruptured Cerebral Aneurysms: Evaluation and Management. ScientificWorldJournal. 2015; 2015 : 954954, doi: 10.1155/2015/954954, indexed in Pubmed: 26146657.

3. Wiebers D. Unruptured intracranial aneurysms: natural history, clinical outcome, and risks of surgical and endovascular treatment. The Lancet. 2003; 362(9378): 103-110, doi: 10.1016/s01406736(03)13860-3.

4. Wakhloo AK, Lylyk P, de Vries J, et al. Surpass Study Group. Surpass flow diverter in the treatment of intracranial aneurysms: a prospective multicenter study. AJNR Am J Neuroradiol. 2015; 36(1): 98-107, doi: 10.3174/ajnr.A4078, indexed in Pubmed: 25125666.

5. Kiyosue H, Tanoue S, et al. Anatomic features predictive of complete aneurysm occlusion can be determined with three-dimensional digital subtraction angiography. American Journal of Neuroradiology. 2002; 23(7): 1206-1213.

6. Tamatani S, Ito Y, Abe H, et al. Evaluation of the stability of aneurysms after embolization using detachable coils: correlation between stability of aneurysms and embolized volume of aneurysms. AJNR Am J Neuroradiol. 2002; 23(5): 762-767, indexed in Pubmed: 12006273.

7. Slob MJ, Sluzewski M, van Rooij WJ. The relation between packing and reopening in coiled intracranial aneurysms: a prospective study. Neuroradiology. 2005; 47(12): 942-945, doi: 10.1007/s00234-0051446-9, indexed in Pubmed: 16136261.

8. Murayama Y, Nien YL, Duckwiler G, et al. Guglielmi detachable coil embolization of cerebral aneurysms: 11 years' experience. J Neurosurg. 2003; 98(5): 959-966, doi: 10.3171/jns.2003.98.5.0959, indexed in Pubmed: 12744354.

9. Alshekhlee A, Mehta S, Edgell RC, et al. Hospital mortality and complications of electively clipped or coiled unruptured intracranial aneurysm. Stroke. 2010; 41(7): 1471-1476, doi: 10.1161/STROKEAHA.110.580647, indexed in Pubmed: 20522817. 
10. Jo KII, Yeon JeY, Kim KHa, et al. Predictors of thromboembolism during coil embolization in patients with unruptured intracranial aneurysm. Acta Neurochir (Wien). 2013; 155(6): 1101-1106, doi: 10.1007/ s00701-013-1706-0, indexed in Pubmed: 23624636.

11. Ihn YK, Shin SH, Baik SK, et al. Complications of endovascular treatment for intracranial aneurysms: Management and prevention. Interv Neuroradiol. 2018; 24(3): 237-245, doi: 10.1177/1591019918758493, indexed in Pubmed: 29466903.

12. McDonald JS, McDonald RJ, Fan J, et al. Comparative effectiveness of unruptured cerebral aneurysm therapies: propensity score analysis of clipping versus coiling. Stroke. 2013; 44(4): 988-994, doi: 10.1161/ STROKEAHA.111.000196, indexed in Pubmed: 23449260.

13. Naggara ON, Lecler A, Oppenheim C, et al. Endovascular treatment of intracranial unruptured aneurysms: a systematic review of the literature on safety with emphasis on subgroup analyses. Radiology. 2012; 263(3): 828-835, doi: 10.1148/radiol.12112114, indexed in Pubmed: 22623696.

14. Spetzler RF, McDougall CG, Zabramski JM, et al. The Barrow Ruptured Aneurysm Trial. J Neurosurg. 2012; 116(1): 135-144, doi: 10.3171/2011.8.JNS101767, indexed in Pubmed: 22054213.

15. Spetzler RF, McDougall CG, Albuquerque FC, et al. The Barrow Ruptured Aneurysm Trial: 3-year results. J Neurosurg. 2013; 119(1): 146-157, doi: 10.3171/2013.3.JNS12683, indexed in Pubmed: 23621600.

16. Spetzler RF, McDougall CG, Zabramski JM, et al. The Barrow Ruptured Aneurysm Trial: 6-year results. J Neurosurg. 2015; 123(3): 609-617, doi: 10.3171/2014.9.JNS141749, indexed in Pubmed: 26115467.

17. Spetzler RF, McDougall CG, Zabramski JM, et al. Ten-year analysis of saccular aneurysms in the Barrow Ruptured Aneurysm Trial. J Neurosurg. 2019 [Epub ahead of print]: 1-6, doi: 10.3171/2018.8.JNS181846, indexed in Pubmed: 30849758.

18. Mooney M, Simon E, Brigeman S, et al. Long-term results of middle cerebral artery aneurysm clipping in the Barrow Ruptured Aneurysm Trial. Journal of Neurosurgery. 2019; 130(3): 895-901, doi: 10.3171/2017.10.jns172183.

19. Zhang Q, Jing L, Liu J, et al. Predisposing factors for recanalization of cerebral aneurysms after endovascular embolization: a multivariate study. J Neurointerv Surg. 2018; 10(3): 252-257, doi: 10.1136/neurintsurg-2017-013041, indexed in Pubmed: 28377443.
20. Jeon JP, Cho YD, Rhim JK, et al. Extended monitoring of coiled aneurysms completely occluded at 6-month follow-up: late recanalization rate and related risk factors. Eur Radiol. 2016; 26(10): 3319-3326, doi: 10.1007/s00330-015-4176-3, indexed in Pubmed: 26747259.

21. Lecler A, Raymond J, Rodriguez-Régent C, et al. Intracranial Aneurysms: Recurrences More than 10 Years after Endovascular Treatment-A Prospective Cohort Study, Systematic Review, and Meta-Analysis. Radiology. 2015; 277(1): 173-180, doi: 10.1148/radiol.2015142496, indexed in Pubmed: 26057784.

22. Sugiyama SI, Niizuma K, Sato K, et al. Blood Flow Into Basilar Tip Aneurysms: A Predictor for Recanalization After Coil Embolization. Stroke. 2016; 47(10): 2541-2547, doi: 10.1161/STROKEAHA.116.013555, indexed in Pubmed: 27625377.

23. Li C, Wang S, Chen J, et al. Influence of hemodynamics on recanalization of totally occluded intracranial aneurysms: a patient-specific computational fluid dynamic simulation study. J Neurosurg. 2012; 117(2): 276-283, doi: 10.3171/2012.5.JNS111558, indexed in Pubmed: 22680247.

24. Gruszka W, Zbroszczyk M, Komenda J, et al. The role of inflammation and potential pharmacological therapy in intracranial aneurysms. Neurol Neurochir Pol. 2018; 52(6): 662-669, doi: 10.1016/j. pjnns.2018.08.002, indexed in Pubmed: 30190209.

25. Nastasovic T, Milakovic B, Stosic M, et al. Predictors of unfavourable outcome in aneurysmal subarachnoid haemorrhage. Neurol Neurochir Pol. 2019; 53(6): 421-427, doi: 10.5603/PJNNS.a2019.0051, indexed in Pubmed: 31657445.

26. Yeon EK, Cho YD, Yoo DH, et al. Delayed Progression to Major Recanalization in Coiled Aneurysms with Minor Recanalization at 36-Month Follow-up : Incidence and Related Risk Factors. Clin Neuroradiol. 2020 [Epub ahead of print], doi: 10.1007/s00062-020-00887-1, indexed in Pubmed: 32144482.

27. Tailor J, Goetz P, Chandrashekar H, et al. Stability of ruptured intracranial aneurysms treated with detachable coils: is delayed follow-up angiography warranted? Br J Neurosurg. 2010; 24(4): 405-409, doi: 10.3109/02688697.2010.487130, indexed in Pubmed: 20632877.

28. Raymond J, Guilbert F, Weill A, et al. Long-term angiographic recurrences after selective endovascular treatment of aneurysms with detachable coils. Stroke. 2003; 34(6): 1398-1403, doi: 10.1161/01. STR.0000073841.88563.E9, indexed in Pubmed: 12775880. 